\title{
Fatigue constrained topology optimization
}

\author{
Erik Holmberg, Bo Torstenfelt and Anders Klarbring
}

\section{Linköping University Post Print}

\section{Tweet}

N.B.: When citing this work, cite the original article.

The original publication is available at www.springerlink.com:

Erik Holmberg, Bo Torstenfelt and Anders Klarbring, Fatigue constrained topology optimization, 2014, Structural and multidisciplinary optimization (Print), (50), 2, 207-219. http://dx.doi.org/10.1007/s00158-014-1054-6

Copyright: Springer Verlag (Germany)

http://www.springerlink.com/?MUD=MP

Postprint available at: Linköping University Electronic Press

http://urn.kb.se/resolve?urn=urn:nbn:se:liu:diva-88093 


\title{
Fatigue constrained topology optimization
}

\author{
Erik Holmberg · Bo Torstenfelt • Anders Klarbring
}

\begin{abstract}
We present a contribution to a relatively unexplored application of topology optimization: structural topology optimization with fatigue constraints. A probability based high-cycle fatigue analysis is combined with principal stress calculations in order to find the topology with minimum mass that can withstand prescribed variable-amplitude loading conditions for a specific life time. This allows us to generate optimal conceptual designs of structural components where fatigue life is the dimensioning factor.

We describe the fatigue analysis and present ideas that make it possible to separate the fatigue analysis from the topology optimization. The number of constraints is kept low as they are applied to stress clusters, which are created such that they give adequate representations of the local stresses. Optimized designs constrained by fatigue and static stresses are shown and a comparison is also made between stress constraints
\end{abstract}

E. Holmberg

Division of Mechanics, Department of Management and Engineering, Institute of Technology, Linköping University,

SE 58183 Linköping, Sweden

E-mail: erik.holmberg@liu.se

Present address:

Saab AB,

SE 58188 Linköping, Sweden

E-mail: erik.holmberg@saabgroup.com

B. Torstenfelt

Division of Solid Mechanics, Department of Management and Engineering, Institute of Technology, Linköping University,

SE 58183 Linköping, Sweden

E-mail: bo.torstenfelt@liu.se

A. Klarbring

Division of Mechanics, Department of Management and Engineering, Institute of Technology, Linköping University,

SE 58183 Linköping, Sweden

E-mail: anders.klarbring@liu.se based on the von Mises criterion and the highest tensile principal stresses.

The paper is written with focus on structural parts in the avionic industry, but the method applies to any load carrying structure, made of linear elastic isotropic material, subjected to repeated loading conditions.

Keywords Topology optimization · Fatigue constraints · Stress constraints · Principal stress . Eigenvalues $\cdot$ Clusters

\section{Introduction}

In this paper we introduce high-cycle fatigue constraints in topology optimization of load carrying structures. Our aim is to achieve a design with minimum mass where both the fatigue life due to repeated loading conditions as well as static stresses are considered. The intended use is in a conceptual design phase, where the general shape of a structural part is sought. Thus, the fatigue and static stress constraints used in this paper are not intended to replace the final dimensioning or to validate the design, but are intended to generate a conceptual design with minimum mass that will not require major modifications in later design phases.

The traditional formulation in topology optimization, where the stiffness is maximized for a prescribed mass, has some advantages, such as computational efficiency and as a tool to find optimal load paths with respect to stiffness. However, the solutions often contain large stress concentrations and will require major design changes in order to fulfil requirements on stress and fatigue. The final design might therefore be far from an optimal minimum mass design.

In topology optimization the finite element method is used to discretize the problem and a design variable 
is connected to each finite element that belongs to the design domain, see e.g. [7]. We want to have a so-called black-and-white final design where the design variables represent holes (white) or solid material (black). Therefore, intermediate design variable values are penalized in order to make them unfavorable in terms of stiffness and stress. We use SIMP (Solid Isotropic Material with Penalization), as suggested by Bendsøe [3], to decrease the stiffness for intermediate design variable values and a similar penalization that increases the stress. The stress penalization was discussed in the qp-approach by Bruggi [4], but it is here used in the form presented by Le et al. [19] and further discussed by Holmberg et al. [15].

A frequently occurring discussion in the literature regarding stress constraints is the singularity phenomenon, i.e. increasing stresses when the design variables approach zero, which prevents holes from being created, see for example Kirsch [18], Rozvany and Birker [29], Guo et al. [13] and Cheng and Guo [6] among others. This problem is avoided with the stress penalization used in this paper, which was shown in Holmberg et al. [15].

Fatigue is a local phenomenon, which implies that fatigue constraints need to be considered in each point. However, local constraints are very expensive in terms of computational time and using a constraint in each stress evaluation point is not an option except for very small problems, see Duysinx and Bendsøe [10]. Therefore, we use a clustered approach as presented in Holmberg et al. [15], which is somewhat similar to the approaches by Le et al. [19] and Paris et al. [26]. In the clustered approach, the stress evaluation points are grouped into a relatively low number of clusters and one constraint is then applied to each cluster, instead of each stress evaluation point.

We use a modified P-norm for creating a clustered stress measure for each cluster. The modification is such that the clustered stress measure approaches the maximum local stress when the clusters are updated based on the current stress level. This stress measure has been used successfully in Holmberg et al. [15] and [16], where non-differentiability due to the cluster update is discussed. A main difference regarding the clustered stress measure in this paper is that we, for reasons discussed in Section 5, use the highest principal stresses instead of von Mises stresses. The choice of clustered stress measure is not crucial for the method developed in this paper, several other techniques for creating a global or clustered stress measure are presented in the literature: París et al. [26] and Wang and Luo [35] use stress measures based on the Kreisselmeier-Steinhauser function, Le et al. [19] use a normalization function which scales a P-norm measure such that it better represents the maximum local stress. Stress based problems are also solved in a level-set framework by Allaire and Jouve [1] and Zhang et al. [36] among others.

Fatigue has been a well known phenomenon for a very long time and scientific reports have been published since the first half of the nineteenth century. However, fatigue constraints have not been used that extensively in structural optimization problems. Some authors that have used structural optimization in order to find designs that fulfil fatigue life aspects are for example Kaya et al. [17], where a failed clutch fork was investigated and compliance based topology optimization followed by shape optimization by the response surface method was used to achieve a design with a lower von Mises stress. The fatigue analysis was made using the software MSC Fatigue [23] and a constant amplitude load curve. Mrzyglod and Zielinski [21] made a shape optimization of a suspension arm with multiaxial highcycle fatigue constraints, using Dang Van's criterion. The authors evaluated different criteria in [22] and discussed the implementation in [20]. A fatigue analysis using the software FEMFAT was integrated into the optimization software TOSCA in [12], where shape and topology optimization was made with fatigue considerations. Desmorat and Desmorat maximized the fatigue life, considering elasto-plastic low-cycle fatigue, in a 3D topology optimization problem in [9]. Fatigue constraints are also available for topology optimization in Optistruct [24].

The motivation for this paper is that topology optimization in conceptual design phases allows for much greater mass reductions than shape and size optimization in later design phases. Introducing fatigue constraints and stress constraints, i.e. requirements that later will be used when the structural part is dimensioned, in the topology optimization, allows for more mature designs that do not have to undergo severe modifications in order to become a final design. This paper will not focus on mechanisms behind the fatigue phenomenon, such as material aspects, the influence of different load ratios etc. We use a fatigue analysis as a tool to find a structure that can endure repeated loading conditions without failure.

The paper is organized as follows: an overview of the considered fatigue methodology is presented in Section 2 and in Section 3 we explain how the fatigue analysis is used in the optimization. The optimization problem is formulated in Section 4 and the clustered stress measure is discussed in Section 5. Derivation of the sensitivity analysis, including a discussion on eigenvalue derivatives, is presented in Section 6. Finally, examples 


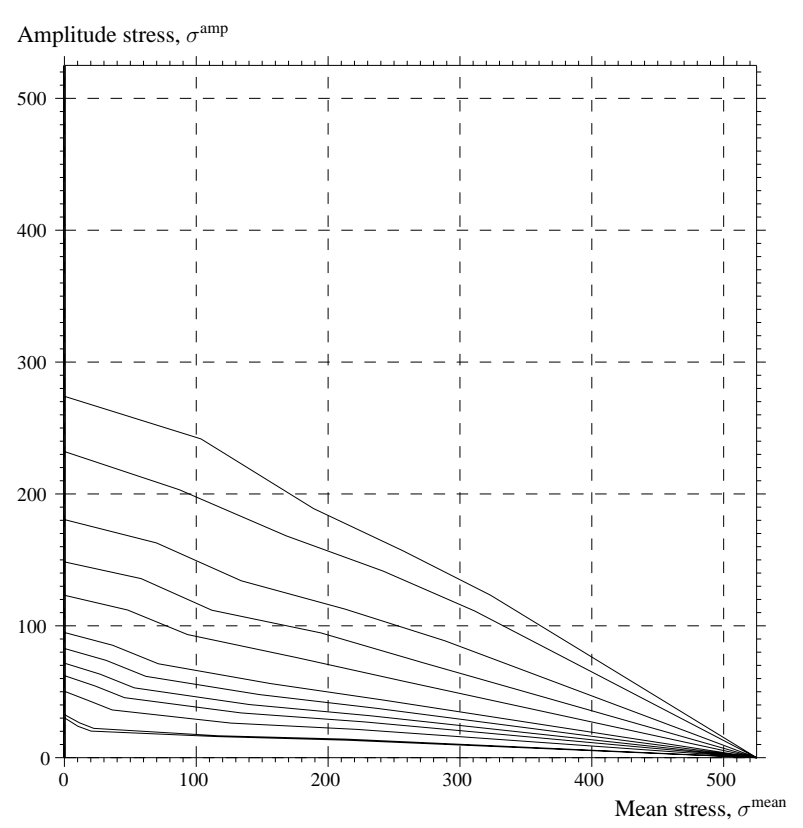

Fig. 1: Haigh diagram which is represented by the operators $\mathbb{H}_{l}$ in (3). The curves correspond to different allowable number of cycles, $N$

are shown in Section 7 and conclusions are drawn in Section 8.

\section{Fatigue analysis}

We use a traditional high-cycle fatigue methodology, see for example Suresh [31] or Dahlberg and Ekberg [8], where the damage for different loads are accumulated using Palmgren-Miner's rule and no distinction is made between crack initiation, crack propagation and fatigue failure. The loads are given by a variable-amplitude load spectrum, as discussed in Section 2.1, and material data is given by Wöhler and Haigh diagrams, which are based on fatigue tests. A Wöhler diagram specifies the number of cycles to fatigue failure as a function of the stress amplitude, i.e. the diagram represents a constant load ratio, $R=F_{\min } / F_{\max }$. A Haigh diagram, see Figure 1, describes the relationship between the mean stress and the stress amplitude for specific number of cycles; thus, it represents a series of Wöhler diagrams for different load ratios.

\subsection{Load spectrum}

When an aircraft is designed, all missions it is intended to fulfil are estimated. For a fighter aircraft, a mission can be for example training, combat or show and all missions are usually flown a large number of times. The

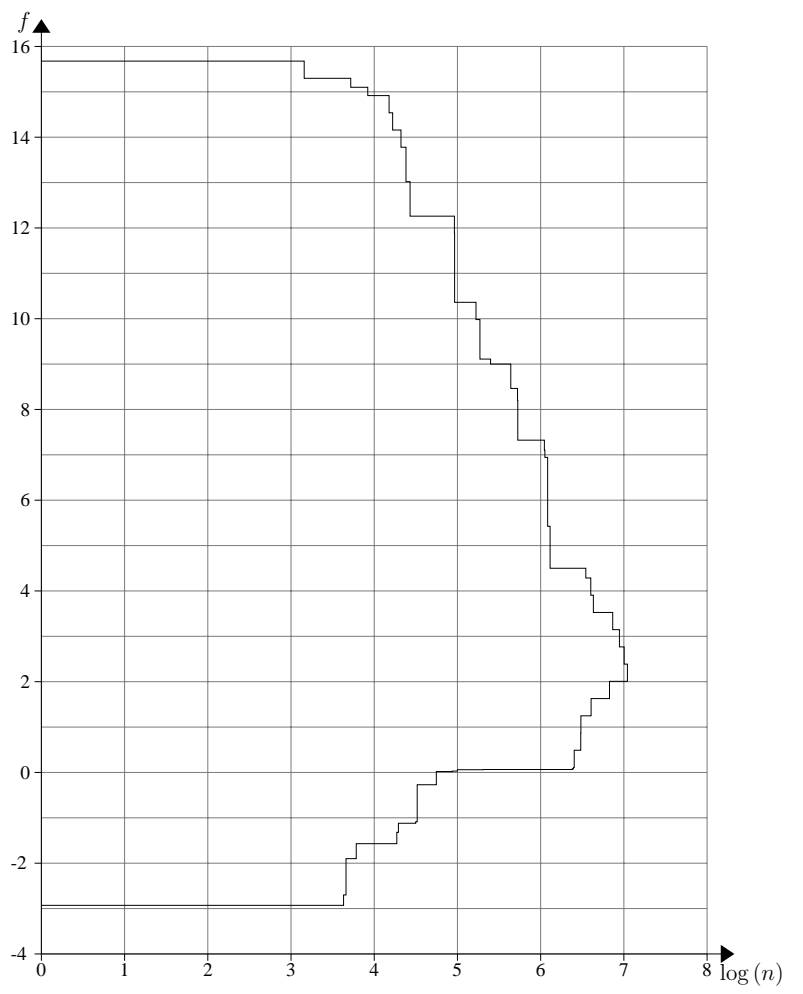

Fig. 2: Load spectrum representing the load factor $f$ on the ordinate and the logarithm of the number of cycles $n$ on the abscissa. It represents the operators $\mathbb{S}_{l}$ in (2) and also provides $n_{l}$

loads on the aircraft during each mission and for its entire life are determined. Local load spectra can then be created, so that all loads for the part that is considered for optimization can be estimated. How the loads are determined is beyond the scope of this paper, we assume that the loads are known and that load pairs have been identified from peak and trough values. An example of a load spectrum is shown in Figure 2. The load spectrum specifies the load factor $f$, the corresponding load at each load level is then $m g f$, where $m$ is the mass and $g$ is the acceleration of gravity. We apply a unit load in the finite element analysis (FE-analysis) and the actual loads for the fatigue analysis are then given by the load spectrum. The same unit load is used for the static stress analysis; the allowable static stress limit is therefore divided by the magnitude of the static load in order to get the corresponding stress limit for a unit load.

\subsection{Damage accumulation at a point}

The fatigue damage at a point of the structure is determined as follows: the stress response for a unit load is determined by an FE-analysis, this can be seen as an 
operator $\mathbb{F} \mathbb{E}$ that maps a unit load $F_{\text {unit }}$ in the current design $\boldsymbol{x}$ to a corresponding stress $\sigma_{\text {unit }}(\boldsymbol{x})$, as

$\sigma_{\text {unit }}(\boldsymbol{x})=\mathbb{F} \mathbb{E}\left(F_{\text {unit }}, \boldsymbol{x}\right)$.

We only consider linear elastic materials, so $\sigma_{\text {unit }}(\boldsymbol{x})$ is linearly scaled by the load levels in the spectrum. The load levels in each load pair $l$ are characterized by the corresponding mean stress $\sigma_{l}^{\text {mean }}(\boldsymbol{x})$ and amplitude stress $\sigma_{l}^{\text {amp }}(\boldsymbol{x})$. This is expressed by operators $\mathbb{S}_{l}$, one for each load pair, written as

$\left(\sigma_{l}^{\text {mean }}(\boldsymbol{x}), \sigma_{l}^{\mathrm{amp}}(\boldsymbol{x})\right)=\mathbb{S}_{l}\left(\sigma_{\text {unit }}(\boldsymbol{x})\right)$.

The load spectrum also gives the number of cycles $n_{l}$ for each load pair, see Figure 2. The allowable number of cycles $N_{l}$ are then determined from a Haigh diagram, by the operators $\mathbb{H}_{l}$, such that

$N_{l}=\mathbb{H}_{l}\left(\sigma_{l}^{\text {mean }}(\boldsymbol{x}), \sigma_{l}^{\text {amp }}(\boldsymbol{x})\right)=\mathbb{H}_{l}\left(\mathbb{S}_{l}\left(\sigma_{\text {unit }}(\boldsymbol{x})\right)\right)$,

where (2) has been used in the second step.

Palmgren-Miner's rule, [31], [8], is then used to calculate the accumulated damage $D$ by comparing the actual number of cycles to the allowable number of cycles for all $L$ load pairs in the spectrum. Thus,

$D\left(\sigma_{\text {unit }}(\boldsymbol{x})\right)=\sum_{l=1}^{L} \frac{n_{l}}{N_{l}}=\sum_{l=1}^{L} \frac{n_{l}}{\mathbb{H}_{l}\left(\mathbb{S}_{l}\left(\sigma_{\text {unit }}(\boldsymbol{x})\right)\right)}$.

Fatigue failure is expected to occur if $D=1$, but generally a lower value is used for safety, i.e. $D \leq \bar{D} \leq 1$, where $\bar{D}$ is the allowable cumulative damage.

\subsection{Fatigue data}

In addition to the load and stress levels, the fatigue life depends on several factors: the surface roughness, the surface treatment and the environment affect the surface of the part and thus the risk of a crack initiation. Further, stress concentration factors, $K_{t}$, have a prominent effect on the fatigue life and the size of the influenced volume, compared to the volume of the test specimen, also has an effect on the expected life.

The allowable number of cycles for different load pairs are determined from (3) using a Haigh diagram, which is based on numerous tests performed on polished test specimens and constructed so that the probability of failure should be below a certain percentage. The diagram is then reduced with respect to the risk of scatter in the material and due to the factors mentioned above, in order to obtain a diagram that corresponds to the state in the actual point of interest. In the topology optimization, we do not want to treat these factors as variables and some simplifications are therefore in order: the surface roughness depends on what type of machining that has been used, and it is assumed to be constant for the whole structure, the surface treatment and the environment it will be subjected to is also likely to be the same for the entire structure and the size influence factor is neglected. For treating stress concentrations, different diagrams are constructed for different $K_{t}$-factors, using notched test specimens. Due to the importance of the $K_{t}$-factor it seems reasonable to try to give at least a rough estimation of it in each stress evaluation point, for example by comparing the lengthwise gradient with the gradient in different notches. However, in this paper we will not put any effort into estimating $K_{t}$-factors. Instead a conservative approach (in the sense that fatigue is less likely to occur) is used; in most relevant structures a fatigue failure occurs at a stress concentration, if we therefore treat all stresses as if they belong to a point where $K_{t}=1$, we know that we are underestimating the fatigue life. This is conservative because for a stress concentration, the volume affected by the higher stress is small, it is therefore a low probability that a material defect exists in that volume. For the same stress level and without a stress concentration, the affected volume is greater and the probability of failure becomes higher. Thus, using the stress from the FE-analysis, that might have been in a stress concentration, together with data for $K_{t}=1$ will be conservative, which is preferable in the conceptual design phase. However, to assume that no stress concentration exists might be too conservative and result in unnecessarily heavy designs. Therefore, instead of $K_{t}=1$, we use material data for $K_{t}=1.5$ in this paper.

With these approaches, the design dependence can be removed from the fatigue analysis, which is then only dependent on the stress. This implies that the fatigue data can be specified prior to the optimization and then considered as constant.

\section{Optimization with respect to fatigue}

We focus our attention on structural parts on a military aircraft, where the loads have been determined and compiled into a load spectrum. The loads are due to masses which are accelerated by manoeuvres, landings, vibrations etc. and the load spectrum contains all loads for the entire life of the aircraft. In the aircraft industry, fatigue life is often expressed in terms of flight hours and an aircraft is designed for a specific number of flight hours. Thus, there is no need to design parts for infinite life. Instead, structural optimization can be used to design the part such that fatigue will not occur 


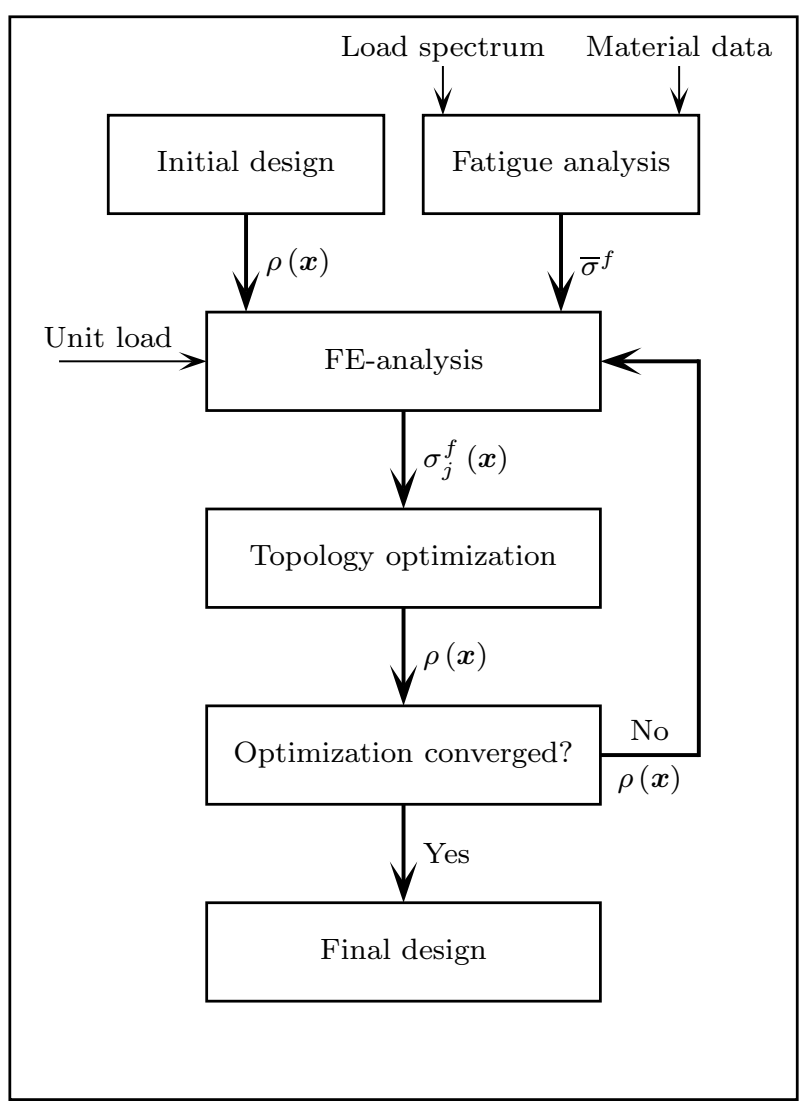

Fig. 3: Flow scheme for fatigue constraints

during the specific finite life, or before predetermined service intervals, i.e. a so-called Safe-Life approach is used.

We note that optimization with respect to infinite life can be made if the allowable fatigue stress is constrained below the endurance limit.

In topology optimization, a relatively coarse mesh and elements with linear shape functions are often used due to computational cost considerations. The stress field calculated from such a mesh is therefore not that accurate and may not be adequate for fatigue analysis. However, as we are designing a structure in the conceptual design stage, the importance lies in avoiding stress concentrations and to size the structural members with respect to the critical fatigue stress. As mentioned in the introduction, the fatigue analysis in the optimization is not intended to replace a final fatigue analysis.

\subsection{Fatigue constraints}

In order to use fatigue life as a constraint in structural optimization, the fact that the part is designed for a specific life time can be used to determine a criterion for fatigue failure.
We perform the fatigue analysis and the topology optimization in two separate steps, which is possible if the fatigue factors are fixed, as discussed in Section 2.3. First, we seek the highest stress value that gives an allowable cumulative damage in (4). This stress, which is not a function of the design, is denoted the critical fatigue stress, $\bar{\sigma}^{f}$ and it is then used as constraint limit in the topology optimization, where we seek the design $\boldsymbol{x}$. That is, we convert the fatigue constraint into a stress constraint. The flow scheme is shown in Figure 3.

The critical fatigue stress for a unit load is found by solving a problem where $\bar{\sigma}^{f}$ is the variable and the objective is to maximize it subjected to a constraint on the cumulative damage in (4). The problem reads

$\left(\mathbb{P}_{\text {crit }}\right)\left\{\begin{array}{l}\max _{\bar{\sigma}^{f}} \bar{\sigma}^{f} \\ \text { s.t. }\left\{\sum_{l=1}^{L} \frac{n_{l}}{\mathbb{H}_{l}\left(\mathbb{S}_{l}\left(\bar{\sigma}^{f}\right)\right)} \leq \bar{D} .\right.\end{array}\right.$

The problem can be solved as an optimization problem or, as $\left(\mathbb{P}_{\text {crit }}\right)$ only needs to be solved once, $\bar{\sigma}^{f}$ can be obtained manually by running the fatigue analysis, (2)(4), for different stress values.

For completeness we note that if fatigue factors, e.g. $K_{t}$-factors, are to be variable in the optimization problem, we may use (4) as constraint function and perform the fatigue analysis in every iteration, between the FE-analysis and the topology optimization in the flow scheme in Figure 3.

\section{Problem formulation}

Our main goal is to find a light weight structure. The objective function is therefore to minimize the total mass, i.e. the sum of the element masses $m_{e}$ scaled by the corresponding filtered variable value $\rho_{e}(\boldsymbol{x})$, which is connected to the design variables through a design variable filter [5]. Using the design variable filter we avoid checkerboard phenomena and mesh dependency of the solution. In the design variable filter, the filtered variable $\rho_{e}(\boldsymbol{x})$ is created by weighting design variable $x_{e}$ with neighbouring design variables, as

$\rho_{e}(\boldsymbol{x})=\frac{\sum_{k \in \Omega_{e}} w_{k} x_{k}}{\sum_{k \in \Omega_{e}} w_{k}}=\sum_{k=1}^{n_{e}} W_{e k} x_{k}$,

where $n_{e}$ is the number of design variables and the set $\Omega_{e}$ contains all the design variables within a given filter radius $r_{0}$, measured from $x_{e}$. The number of nonzero coefficients in $W_{e k}$ for a particular $e$ will be the same as the number of members in $\Omega_{e}$. The weight factor 
$w_{k}$ is linearly decreased with $r_{k}$, which is the distance between the centroid of design variable $x_{k}$ and $x_{e}$, thus

$w_{k}=\frac{r_{0}-r_{k}}{r_{0}}$

The filtered variables $\boldsymbol{\rho}(\boldsymbol{x})$ are considered to be physical variables in the sense that they define the mass and the stiffness, where the latter is penalized using SIMP [3]. That is, we have a penalization function, $\eta_{K}\left(\rho_{e}(\boldsymbol{x})\right)=\left(\rho_{e}(\boldsymbol{x})\right)^{q}$, that scales the solid material expanded element stiffness matrices $\boldsymbol{K}_{e}$. The penalization factor $q>1$ is usually, and in this paper, set to $q=3$. The global stiffness matrix $\boldsymbol{K}(\boldsymbol{\rho}(\boldsymbol{x}))$ then becomes

$\boldsymbol{K}(\boldsymbol{\rho}(\boldsymbol{x}))=\sum_{e=1}^{n_{e}} \eta_{K}\left(\rho_{e}(\boldsymbol{x})\right) \boldsymbol{K}_{e}$.

The equilibrium equation needs to be satisfied in each optimization iteration. It reads

$\boldsymbol{K}(\boldsymbol{\rho}(\boldsymbol{x})) \boldsymbol{u}=\boldsymbol{F}$,

where $\boldsymbol{u}$ is the vector of nodal displacements and $\boldsymbol{F}$ is a vector of prescribed external unit loads. We use a nested formulation where the displacement vector is solved for in the FE-analysis and thus is considered a known function of the design variables, i.e.

$\boldsymbol{u}=\boldsymbol{u}(\boldsymbol{x})=\boldsymbol{K}^{-1}(\boldsymbol{\rho}(\boldsymbol{x})) \boldsymbol{F}$.

In addition to the fatigue constraint, a static stress constraint based on the von Mises criterion is used, as described by Holmberg et al. in [15]. The problem formulation reads

$$
\left\{\begin{array}{l}
\min _{\boldsymbol{x}} \sum_{e=1}^{n_{e}} m_{e} \rho_{e}(\boldsymbol{x}) \\
\text { s.t. }\left\{\begin{array}{l}
\sigma_{j}^{f}(\boldsymbol{x}) \leq \bar{\sigma}^{f}, j=1, . ., n_{f c} \\
\sigma_{i}^{s}(\boldsymbol{x}) \leq \bar{\sigma}^{s}, i=1, . ., n_{s c} \\
\epsilon \leq x_{e} \leq 1, e=1, . ., n_{e},
\end{array}\right.
\end{array}\right.
$$

where $n_{f c}$ is the number of fatigue constraints and $n_{s c}$ is the number of static stress constraints. A small number $\epsilon>0$ is used as lower bound on the design variables in order to keep the stiffness matrix positive definite in (6). The allowable static stress is $\bar{\sigma}^{s}$ and the corresponding static stress measure for constraint $i$ is denoted $\sigma_{i}^{s}(\boldsymbol{x})$. The fatigue stress measure $\sigma_{j}^{f}(\boldsymbol{x})$ is defined in Section 5 and the critical fatigue stress $\bar{\sigma}^{f}$ is obtained by solving $\left(\mathbb{P}_{\text {crit }}\right)$.

\section{Clustered fatigue stress measure}

In this section we derive and discuss the fatigue stress measure $\sigma_{j}^{f}(\boldsymbol{x})$ that is used in this paper. We use the same clustered approach that is used for static stress constraints in Holmberg et al. [15], where a modified Pnorm is used to cluster the stresses from several stress evaluation points to one value, which is then constrained.

Fatigue cracks are mainly initiated by shear stresses and propagated by normal stresses. Crack initiation is a phenomenon that usually occurs at the surface since the highest stresses usually occur at the surface and as it is subjected to environmental wear and might have a rough finish. In this paper we consider $2 \mathrm{D}$ structures in plane stress, which have a uniaxial stress state at the boundaries. However, in a topology optimization problem, the boundaries are not well defined as the structure is obtained iteratively and as the boundaries are blurred due to the filter. Therefore, all stress evaluation points contribute to the clustered fatigue stress measures and we use the highest principal stress in each point as stress measure. Further, as fatigue generally does not occur for compressive stresses, only the tensile stresses contribute to the clustered fatigue stress measures.

The stress in each stress evaluation point is penalized based on the value of the corresponding filtered design variable, where the stress is increased for intermediate design variable values. The penalization function

$\eta_{S}\left(\rho_{e}(\boldsymbol{x})\right)=\left(\rho_{e}(\boldsymbol{x})\right)^{\frac{1}{2}}$,

was suggested in [19] and used also in [15], where it proved to work well. The same stress penalization function is used in this paper and it is scaling the stress vector which is calculated in the FE-analysis. The expression for the penalized stress vector reads

$\boldsymbol{\sigma}_{a}(\boldsymbol{x})=\left(\rho_{e}(\boldsymbol{x})\right)^{\frac{1}{2}} \boldsymbol{E} \boldsymbol{B}_{a} \boldsymbol{u}(\boldsymbol{x})$,

where $\boldsymbol{E}$ is the constitutive matrix and $\boldsymbol{B}_{a}$ is the straindisplacement matrix corresponding to stress evaluation point $a$. The stress penalization is a function of $\rho_{e}(\boldsymbol{x})$, which is the filtered variable corresponding to the element to which stress evaluation point $a$ belongs. An element can have several stress evaluation points but only one design variable.

We denote the highest tensile principal stress at a point $a$ by $\sigma_{a}^{1}(\boldsymbol{x}) \geq 0$ and the clustered fatigue stress measure for constraint $j$ is then

$\sigma_{j}^{f}(\boldsymbol{x})=\left(\frac{1}{N_{j}} \sum_{a \in \Omega_{j}}\left(\sigma_{a}^{1}(\boldsymbol{x})\right)^{p}\right)^{\frac{1}{p}}$, 
where $p$ is the P-norm factor, $\Omega_{j}$ is the set of stress evaluation points that belong to cluster $j$ and $N_{j}$ is the number of such points.

It was shown in [15] that it is of great importance how the distribution of stress evaluation points into the clusters is made. Two approaches for the distribution: the Stress level approach and the Distributed stress approach were evaluated, as well as the influence of reclustering frequency. Based on the experience from [15] we use the Stress level approach, where each stress evaluation point is clustered together with other points that have a similar stress level, and we update the clusters at every iteration.

\section{Sensitivity analysis}

We use the method of moving asymptotes (MMA), developed by Svanberg [32], to solve the optimization problem. MMA is a gradient based solver, so we need to calculate the gradient of the objective function and the constraints. The gradient of the objective function and the von Mises based stress constraints were derived by Holmberg et al. [15] and the gradient of the critical fatigue stress constraint in $(\mathbb{P})$ is derived in this section.

We start from the first order derivative of the cumulative damage (4), based on the fatigue stress measure (9), with respect to design variable $x_{b}$. It reads

$\frac{\partial D\left(\sigma_{j}^{f}\right)}{\partial x_{b}}=\frac{\partial D\left(\sigma_{j}^{f}\right)}{\partial \sigma_{j}^{f}} \frac{\partial \sigma_{j}^{f}(\boldsymbol{x})}{\partial x_{b}}$,

where we can identify the first factor as the gradient required when solving $\left(\mathbb{P}_{\text {crit }}\right)$ and the second factor as the gradient required when solving $(\mathbb{P})$, which is derived in the following. The derivation is therefore applicable both for constraints based directly on (4), as well as clustered (fatigue-based) stress constraints based on the highest tensile principal stress.

\subsection{Derivative of the clustered fatigue stress measure}

The gradient of (9) with respect to design variable $x_{b}$ reads

$$
\begin{aligned}
\frac{\partial \sigma_{j}^{f}(\boldsymbol{x})}{\partial x_{b}} & =\sum_{a \in \Omega_{j}} \frac{\partial \sigma_{j}^{f}(\boldsymbol{x})}{\partial \sigma_{a}^{1}} \frac{\partial \sigma_{a}^{1}(\boldsymbol{x})}{\partial x_{b}} \\
& =\sum_{a \in \Omega_{j}} \frac{\partial \sigma_{j}^{f}(\boldsymbol{x})}{\partial \sigma_{a}^{1}} \sum_{j=1}^{n_{e}} \frac{\partial \sigma_{a}^{1}(\boldsymbol{x})}{\partial \rho_{j}} \frac{\partial \rho_{j}(\boldsymbol{x})}{\partial x_{b}} .
\end{aligned}
$$

In (11), the $\partial \sigma_{j}^{f}(\boldsymbol{x}) / \partial \sigma_{a}^{1}$ derivative is calculated as

$$
\frac{\partial \sigma_{j}^{f}(\boldsymbol{x})}{\partial \sigma_{a}^{1}}=\left(\frac{1}{N_{j}} \sum_{a \in \Omega_{j}}\left(\sigma_{a}^{1}(\boldsymbol{x})\right)^{p}\right)^{\left(\frac{1}{p}-1\right)} \frac{1}{N_{j}}\left(\sigma_{a}^{1}(\boldsymbol{x})\right)^{p-1}
$$

and the $\partial \sigma_{a}^{1}(\boldsymbol{x}) / \partial x_{b}$-part indicates that we need to calculate the sensitivity of an eigenvalue.

\subsection{Eigenvalue derivative}

In Section 6.2 - 6.3 we use Einstein's summation convention and we neglect writing the design dependence as well as the index corresponding to the stress evaluation point.

The principal stresses are the eigenvalues of the stress tensor:

$\boldsymbol{S}=\left[\begin{array}{lll}S_{11} & S_{12} & S_{13} \\ S_{12} & S_{22} & S_{23} \\ S_{13} & S_{23} & S_{33}\end{array}\right]$

The stress tensor is symmetric, which implies that its eigenvalues are real. The eigenvalue problem in one stress evaluation point is formulated as

$\left(S_{i j}-\lambda^{\alpha} I_{i j}\right) \phi_{j}^{\alpha}=0$,

where $\lambda^{\alpha}$ are the eigenvalues and $\phi_{j}^{\alpha}$ the components of the corresponding eigenvector. Note that $\alpha$ is not a summation index, but an index to separate the three solutions of the eigenvalue problem.

Derivatives of eigenvalues are discussed in the literature for optimization with respect to eigenfrequencies, see Haftka et al. [14], Seyranian et al. [30], Pedersen [27] and Pedersen and Nielsen [28] among others. It is noted that multiple eigenvalues might occur and that the eigenvectors are not unique for that case, as any linear combination of the two corresponding eigenvectors will satisfy (13). The sensitivity analysis of multiple eigenvalues in structural optimization is discussed by e.g. Seyranian et al. [30] and Pedersen and Nielsen [28]. We also note that, as the design changes, the eigenvectors change and the eigenvalues might then switch position. Thus, the highest eigenvalue in the current iteration might correspond to a different vibration mode, or in our case, a different stress direction, than in the previous iteration. However, eigenfrequency constraints are global in the sense that they are related to the entire structure, while the highest principal stress is calculated in each stress evaluation point and then gives a contribution to the clustered stress measure. In the case of principal stresses, we therefore expect the influence of 
occasional switches and multiple eigenvalues to be negligible. No attempt will therefore be made to handle the fact that a switch in the principal values might occur, nor the fact that two eigenvalues might be identical. In fact, we also note that no multiple eigenvalues actually occurred in the examples in this paper.

Differentiating (13) with respect to design variable $x_{b}$ gives (see Appendix)

$\frac{\partial \lambda^{\alpha}}{\partial x_{b}}=\phi_{i}^{\alpha} \frac{\partial S_{i j}}{\partial x_{b}} \phi_{j}^{\alpha}$

\subsection{Derivative of the highest tensile principal stress}

In (11), we are only interested in the highest tensile principal stress. Thus, $\sigma^{1}=\max \left(\lambda^{1}, 0\right)$, where $\lambda^{1}>$ $\lambda^{2}>\lambda^{3}$ and the corresponding eigenvector components $\phi_{j}^{(1)}$ are from here on denoted $\phi_{j}$. We can then write (14) for the highest tensile principal stress as

$\frac{\partial \sigma^{1}}{\partial x_{b}}=\phi_{i} \frac{\partial S_{i j}}{\partial x_{b}} \phi_{j}$.

However, Voigt notation is used in order to calculate the stresses, meaning that the symmetric stress tensor is replaced by a vector with six components. Equation (15) is therefore rewritten so that the stresses are expressed in Voigt notation. This is done by the use of a transformation vector $\boldsymbol{\Lambda}$ and the Voigt stress vector in (8) as,

$\phi_{i} \frac{\partial S_{i j}}{\partial x_{b}} \phi_{j}=\Lambda_{t} \frac{\partial \sigma_{t}}{\partial x_{b}}$.

Evaluating the left hand side of (16) and using symmetry of $S_{i j}$ we find that $\boldsymbol{\Lambda}$ can be identified as

$\boldsymbol{\Lambda}=\left(\phi_{1}^{2}, \phi_{2}^{2}, \phi_{3}^{2}, 2 \phi_{1} \phi_{2}, 2 \phi_{2} \phi_{3}, 2 \phi_{1} \phi_{3}\right)^{T}$.

The transformation vector (17) and equation (16) can now be used to write (15) in matrix form for stress evaluation point $a$ as

$\frac{\partial \sigma_{a}^{1}(\boldsymbol{x})}{\partial x_{b}}=\boldsymbol{\Lambda}_{a}^{T} \frac{\partial \boldsymbol{\sigma}_{a}(\boldsymbol{x})}{\partial x_{b}}$.

In (18) and through the rest of the paper, no summation indices are used.

\subsection{Stress vector and its derivative}

The stress vector $\boldsymbol{\sigma}_{a}(\boldsymbol{x})$ is defined as the stress vector in solid material penalized by a stress penalization function $\eta_{S}\left(\rho_{e}(\boldsymbol{x})\right)$ as described by (8). The derivative of (8) with respect to design variable $x_{b}$ can now be determined as required in (18). The chain rule is applied since the design variables are filtered in (5):

$$
\begin{aligned}
\frac{\partial \boldsymbol{\sigma}_{a}(\boldsymbol{x})}{\partial x_{b}} & =\frac{\partial \eta_{S}\left(\rho_{e}\right)}{\partial \rho_{e}} \frac{\partial \rho_{e}(\boldsymbol{x})}{\partial x_{b}} \boldsymbol{E} \boldsymbol{B}_{a} \boldsymbol{u}(\boldsymbol{x}) \\
+ & \eta_{S}\left(\rho_{e}(\boldsymbol{x})\right) \boldsymbol{E} \boldsymbol{B}_{a} \sum_{r=1}^{n_{e}}\left(\frac{\partial \boldsymbol{u}(\boldsymbol{x})}{\partial \rho_{r}} \frac{\partial \rho_{r}(\boldsymbol{x})}{\partial x_{b}}\right),
\end{aligned}
$$

where the derivative of the filtered variable with respect to a design variable is the filter weight, $\partial \rho_{e}(\boldsymbol{x}) / \partial x_{b}=$ $W_{e b}$ and $\partial \rho_{r}(\boldsymbol{x}) / \partial x_{b}=W_{r b}$, as defined in (5).

\subsection{Aggregation into (11)}

If (19) is inserted into (18), which is then inserted into (11) together with (12) we get

$$
\begin{aligned}
& \frac{\partial \sigma_{j}^{f}(\boldsymbol{x})}{\partial x_{b}}=\sum_{a \in \Omega_{j}} \frac{\partial \sigma_{j}^{f}(\boldsymbol{x})}{\partial \sigma_{a}^{1}} \frac{\partial \sigma_{a}^{1}(\boldsymbol{x})}{\partial x_{b}} \\
& =\sum_{a \in \Omega_{j}}\left\{\left(\frac{1}{N_{j}} \sum_{a \in \Omega_{j}}\left(\sigma_{a}^{1}(\boldsymbol{x})\right)^{p}\right)^{\left(\frac{1}{p}-1\right)} \frac{1}{N_{j}}\left(\sigma_{a}^{1}(\boldsymbol{x})\right)^{p-1}\right. \\
& \times \boldsymbol{\Lambda}_{a}^{T}\left(\frac{\partial \eta_{S}\left(\rho_{e}\right)}{\partial \rho_{e}} \frac{\partial \rho_{e}(\boldsymbol{x})}{\partial x_{b}} \boldsymbol{E} \boldsymbol{B}_{a} \boldsymbol{u}(\boldsymbol{x})\right. \\
& \left.\left.+\eta_{S}\left(\rho_{e}(\boldsymbol{x})\right) \boldsymbol{E} \boldsymbol{B}_{a} \sum_{r=1}^{n_{e}}\left(\frac{\partial \boldsymbol{u}(\boldsymbol{x})}{\partial \rho_{r}} \frac{\partial \rho_{r}(\boldsymbol{x})}{\partial x_{b}}\right)\right)\right\} .
\end{aligned}
$$

\subsection{Adjoint method}

Due to the clustering, the number of constraints will be less than the number of design variables. Therefore, the adjoint method is preferable for solving (20).

The derivative of the displacements with respect to the design variables is calculated by taking the derivative of the global state equation (7) at a design $\boldsymbol{\rho}(\boldsymbol{x})$ :

$\frac{\partial \boldsymbol{K}(\boldsymbol{\rho}(\boldsymbol{x}))}{\partial x_{b}} \boldsymbol{u}(\boldsymbol{x})+\boldsymbol{K}(\boldsymbol{\rho}(\boldsymbol{x})) \frac{\partial \boldsymbol{u}(\boldsymbol{x})}{\partial x_{b}}=\frac{\partial \boldsymbol{F}}{\partial x_{b}}$.

Applying the chain rule, using $\frac{\partial \boldsymbol{F}}{\partial x}=0$ and rearranging gives

$$
\begin{aligned}
& \sum_{r=1}^{n_{e}}\left(\frac{\partial \boldsymbol{u}(\boldsymbol{x})}{\partial \rho_{r}} \frac{\partial \rho_{r}(\boldsymbol{x})}{\partial x_{b}}\right) \\
& =-\boldsymbol{K}^{-1}(\boldsymbol{\rho}(\boldsymbol{x}))\left[\sum_{r=1}^{n_{e}} \frac{\partial \boldsymbol{K}(\boldsymbol{\rho}(\boldsymbol{x}))}{\partial \rho_{r}} \frac{\partial \rho_{r}(\boldsymbol{x})}{\partial x_{b}} \boldsymbol{u}(\boldsymbol{x})\right]
\end{aligned}
$$


Inserting (21) into (20), the final expression reads

$\frac{\partial \sigma_{j}^{f}(\boldsymbol{x})}{\partial x_{b}}=$

$\sum_{a \in \Omega_{j}}\left(\frac{1}{N_{j}} \sum_{a \in \Omega_{j}}\left(\sigma_{a}^{1}(\boldsymbol{x})\right)^{p}\right)^{\left(\frac{1}{p}-1\right)} \frac{1}{N_{j}}\left(\sigma_{a}^{1}(\boldsymbol{x})\right)^{p-1}$

$\times \boldsymbol{\Lambda}_{a}^{T} \frac{\partial \eta_{S}\left(\rho_{e}\right)}{\partial \rho_{e}} \frac{\partial \rho_{e}(\boldsymbol{x})}{\partial x_{b}} \boldsymbol{E} \boldsymbol{B}_{a} \boldsymbol{u}(\boldsymbol{x})$

$-\boldsymbol{\lambda}_{j}^{T}\left[\sum_{r=1}^{n_{e}} \frac{\partial \boldsymbol{K}(\boldsymbol{\rho}(\boldsymbol{x}))}{\partial \rho_{r}} \frac{\partial \rho_{r}(\boldsymbol{x})}{\partial x_{b}} \boldsymbol{u}(\boldsymbol{x})\right]$,

where the adjoint variable $\boldsymbol{\lambda}_{j}$ is obtained by solving

$\boldsymbol{K}(\boldsymbol{\rho}(\boldsymbol{x})) \boldsymbol{\lambda}_{j}=\sum_{a \in \Omega_{j}} \boldsymbol{B}_{a}^{T} \boldsymbol{E}^{T} \boldsymbol{\Lambda}_{a} \eta_{S}\left(\rho_{e}(\boldsymbol{x})\right) \frac{\partial \sigma_{j}^{f}(\boldsymbol{x})}{\partial \sigma_{a}^{1}}$.

\section{Examples}

In this section we show some designs obtained using static stress and fatigue life constraints and we also show and discuss the difference between stress constraints based on von Mises stresses and stress constraints based on the highest tensile principal stresses. An in-house fatigue code from Saab AB [2] is used for the fatigue analysis, i.e. in order to handle (2), (3) and (4). The fatigue constraints presented in this paper and the static stress constraints in [15] are implemented into the FEprogram TRINITAS [34], in which all analyses, except the fatigue analysis, are done and from which the pictures of the final designs are taken. The Stress level clustering technique, described in [15], is used and the clusters are updated every iteration. The P-norm exponent in (9) is chosen as $p=12$ in all examples and the move limits in MMA have been modified to be more conservative compared to the suggested values in [33]. All designs are initiated from an equal distribution of $\rho_{e}(\boldsymbol{x})=0.5$. The figures show the filtered variables $\boldsymbol{\rho}(\boldsymbol{x})$, where black represents solid material, $\rho_{e}(\boldsymbol{x})=1$, and white represents voids, $\rho_{e}(\boldsymbol{x})=\epsilon$. The stress plots represent the penalized von Mises stresses and should be viewed in colour.

A typical aircraft aluminium is used as design material. The material data is Young's modulus 71000 $\mathrm{MPa}$, density $2.8 \times 10^{-9} \mathrm{ton} / \mathrm{mm}^{3}$, Poisson's ratio 0.33 and yield limit $350 \mathrm{MPa}$. The yield limit is used to determine the static stress constraint limit.

Two test examples are shown, the first is the Lshaped beam which is a popular test example for stress constrained problems, as used by e.g. Le et al. [19], Duysinx and Bendsøe [10], Duysinx and Sigmund [11], Paris et al. [25] and Holmberg et al. [15]. The design

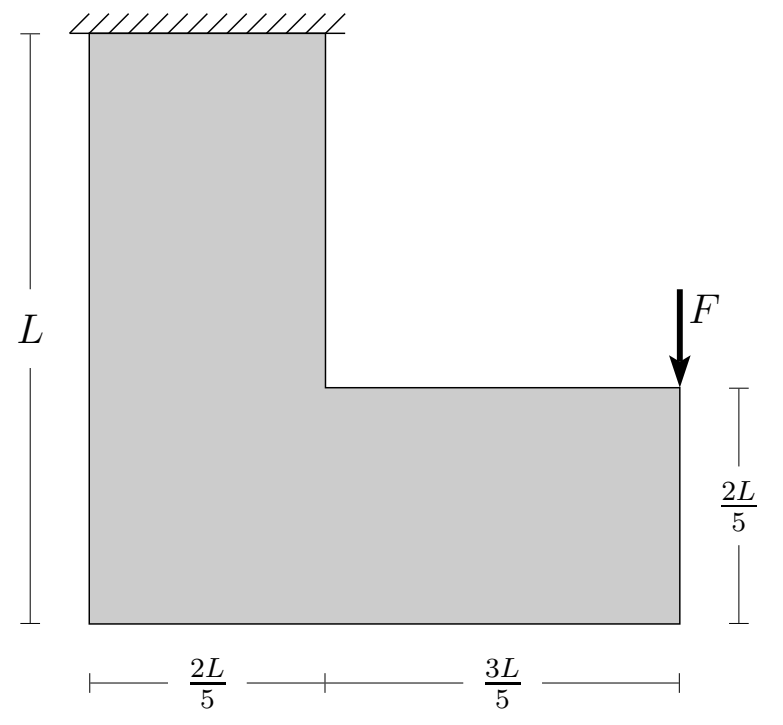

Fig. 4: The L-beam problem

domain, boundary conditions and dimensions of the Lshaped beam are seen in Figure 4, where $L=200 \mathrm{~mm}$ and the thickness is $1 \mathrm{~mm}$. We use 6400 , equal sized, four-node bilinear elements and evaluate the stress in the centroid of each element. A design variable filter is used with the radius $r_{0}=1.5 \times$ element length. In industrial applications, the design domain should be simple to set up and to mesh, the internal corner, which might be due to clearance, is therefore kept in the design domain. This is challenging as an initial stress singularity exists, which cause the optimization to start from a design that is far from feasible and as the singularity needs to be removed in the final design.

The second example is the MBB-beam, which is meshed with 4800 elements. Symmetry is used and the right half of the beam is modelled, as shown in Figure 5 , where $L=100 \mathrm{~mm}$ and the thickness is $1 \mathrm{~mm}$. A design variable filter with the radius $r_{0}=2.0 \times$ element length is used for the MBB-beam. For both examples, the elements in the vicinity of the applied load are solid and not part of the design domain.

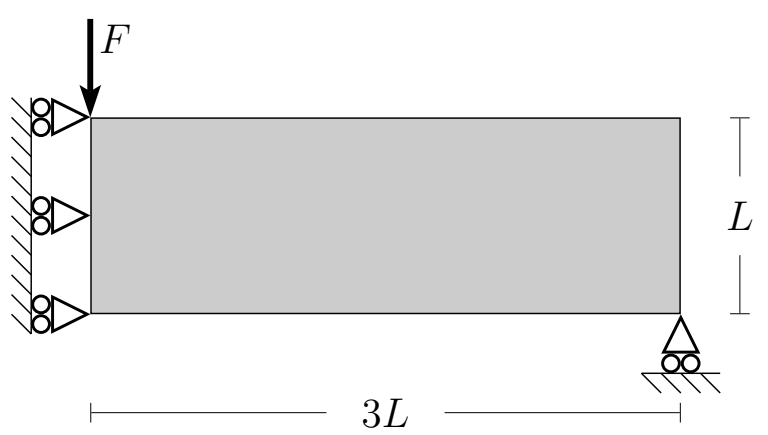

Fig. 5: The MBB problem 
7.1 Applied loads and constraint limits

The load $\boldsymbol{F}$ is due to acceleration of a piece of equipment that is attached to the structure which is to be optimized. The mass of the equipment is $4 \mathrm{~kg}$ and we assume that one load direction is dominating, thus only one load direction is used in the examples. The load factor $f$ is given by the load spectrum shown in Figure 2 . The load spectrum is fictitious in the sense that it is made up for this paper, but it is an adequate representation of accelerations in a certain direction and at a certain position of a fighter aircraft.

The material data for the fatigue analysis is taken from the material library at Saab AB and the Haigh diagram used for the examples is shown in Figure 1. As the material data is confidential, the values of the constant life curves and the reduction factors are not displayed. For the same reason, the cumulative damage limit $\bar{D}$ is not specified. However, in this paper the data is used only to determine the critical fatigue stress $\bar{\sigma}^{f}$, which is presented in the paper.

By solving $\left(\mathbb{P}_{\text {crit }}\right)$ we find that the highest stress, such that $D \leq \bar{D}$, is $\sigma_{\max } \approx 0.129 \mathrm{MPa}$, when material data for $K_{t}=1.5$ is used. The critical fatigue stress $\bar{\sigma}^{f}$, then becomes

$\bar{\sigma}^{f}=K_{t} \sigma_{\max }$,

which is approximately $\bar{\sigma}^{f}=0.194 \mathrm{MPa}$.

For the static stress constraint, a maximum static load, $F_{\text {max }}^{s}=1500 \mathrm{~N}$, is assumed. As a unit load is used in the FE-analysis, the allowable stress to avoid yield is scaled according to

$\bar{\sigma}^{s}=\frac{\sigma_{y i e l d}}{F_{\max }^{s}}$,

which is approximately $\bar{\sigma}^{s}=0.233 \mathrm{MPa}$.

\subsection{Constraints on fatigue life and static stress}

This section presents the L-shaped beam and the MBBbeam, optimized using $(\mathbb{P})$ with fatigue constraints based on the highest tensile principal stresses and static stress constraints based on the von Mises stress criterion. The loads as well as the stress and fatigue limits were described in Section 7.1. The number of clusters that are used influence the computational time and the accuracy of local stresses, more clusters will make the clustered stress measures better approximations of the local stresses, but increase the computational time. The Lbeam is here optimized with seven fatigue constraints and seven static stress constraints.

The topology of the optimized L-beam is seen in Figure 6a. A radius is created in order to avoid a large stress concentration in the internal corner. The optimization also tries to maximize the distance between the two vertical structural components, as the force couple in these parts is then smaller, thus allowing the two components to be thinner, i.e. lighter. The radius in the design in Figure 6a cuts the corner such that the right vertical component is as far to the right as possible and the stresses in the radius, with this discretization, are sufficiently low to satisfy the constraints. We note that different combinations of the $\mathrm{P}$-norm exponent $p$ in (9) and the number of clusters may result in slightly different designs, where the right vertical component sometimes is moved away from the boundary in order to allow for a smoother radius. The left vertical component is loaded in compression and thus sized with respect to the static stress limit $\bar{\sigma}^{s}$, the right is loaded in tension and thus sized with respect to $\bar{\sigma}^{f}$, as $\bar{\sigma}^{f}<\bar{\sigma}^{s}$. A stress plot of the internal corner and parts of the vertical components is given in Figure $6 \mathrm{~b}$.

For the MBB-beam we use five fatigue constraints and five static stress constraints, the optimized design is seen in Figure 7a and the stress plot is seen in Figure 7b. Again, a simple design is obtained, where the structural parts are sized with respect to $\bar{\sigma}^{f}$ and $\bar{\sigma}^{s}$ and stress concentrations are avoided.

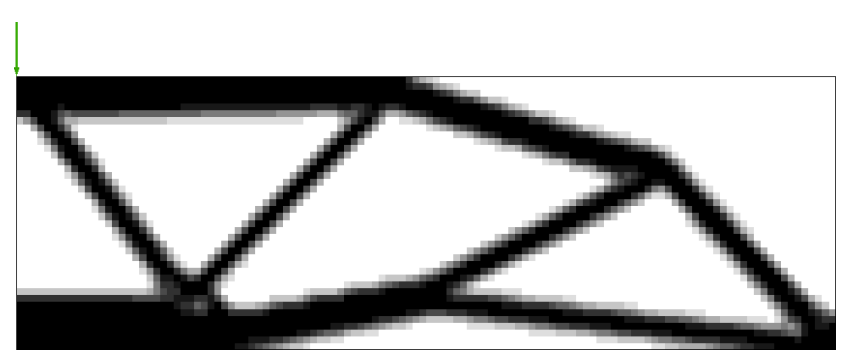

(a) Optimized design

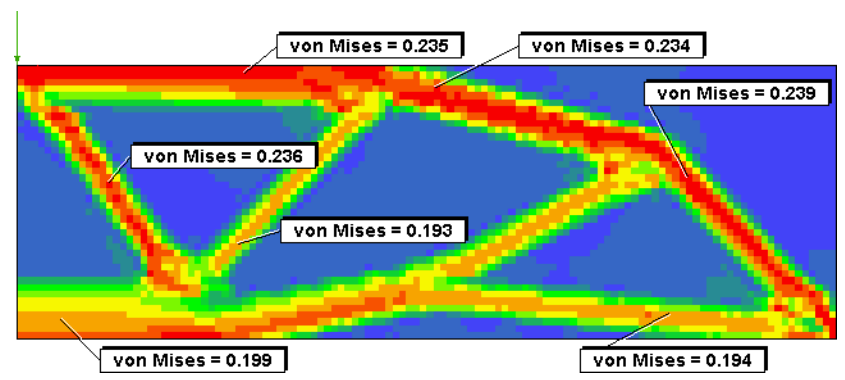

(b) Stress plot with the same color scale as in Figure $6 \mathrm{~b}$

Fig. 7: MBB-beam optimized for minimum mass, with respect to fatigue life and static stress 


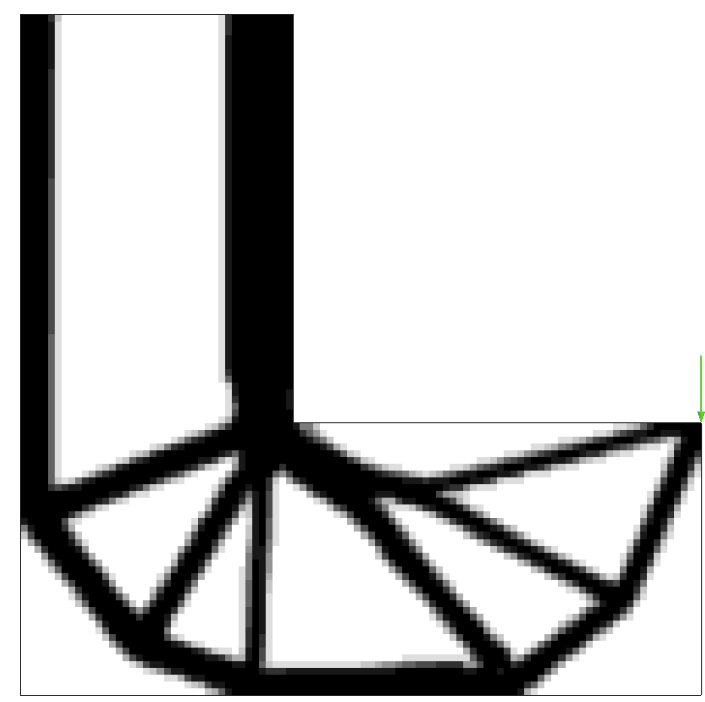

(a) Optimized design

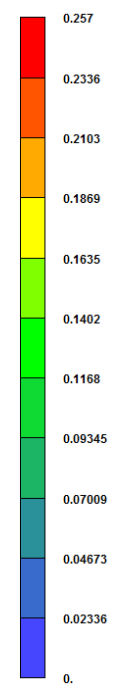

(b) Stress plot, close-up

Fig. 6: L-shaped beam optimized for minimum mass, with respect to fatigue life and static stress

7.3 Comparison between stress constraints based on von Mises or highest tensile principal stress

In this section we compare static stress constraints based on two different stress formulations: the von Mises formulation which is described by Holmberg et al. [15] and the highest tensile principal stresses, derived as a part of the fatigue constraint in this paper. As only the tensile stresses are considered in the principal stress constraints, the stress evaluation points that in the current iteration only have compressive stresses do not contribute to the clustered stress measures. Figure 8 shows the L-shaped beam optimized using formulation $(\mathbb{P})$ without the fatigue constraint. The clustered stress measure has in Figure 8 a been created based on the highest tensile principal stresses, in Figure $8 \mathrm{~b}$ it is based on the von Mises stresses and Figure $8 \mathrm{c}$ is obtained using both stress measures simultaneously. The stress limit is $\bar{\sigma}^{s}=350 \mathrm{MPa}$ and the applied load is $F^{s}=1500$ N. Seven clusters are used for the von Mises based constraint as well as for the principal stress constraint, the number of constraints for a) and b) is thus seven, while it is fourteen for $\mathrm{c}$ ).

For the optimized designs, the structural parts loaded only by compressive forces in Figure 8 a are very thin and the compressive stresses become very high. This is because the mass is minimized and these parts are used only to balance the structure. Additional constraints such as a buckling constraint, a constraint on compressive stresses or alternatively von Mises stresses would be necessary in order to generate a reasonable design. However, this solution is shown here for comparison purposes and as it clearly shows the characteristics of constraints which are based on tensile stresses. Obviously, a problem where the entire structure is loaded by compressive forces cannot be solved with this formulation. The stresses for the designs in Figure 8b and $8 \mathrm{c}$ are close to the stress limit in major parts of the structures, and only a number of local spots have too high stresses which will require additional work in later design phases.

\section{Conclusions}

We have combined fatigue software with clustered principal stress constraints in order to generate an optimized design with minimum mass that can withstand prescribed loading conditions during the service life. We have given an overview of the considered high-cycle fatigue analysis and we have presented ideas that make it possible to separate the fatigue analysis and the topology optimization. We have discussed the clustered approach to reduce the number of constraints and we have presented a sensitivity analysis of the fatigue constraints, which include the sensitivity analysis of principal stresses. Some simple examples involving only one load direction are shown, in which different stress criteria are compared and final designs including fatigue and static stress constraints are shown. The final designs are free from large stress concentrations and are dimensioned with respect to the static and fatigue stress limits. Due to the clustered stress measure, local stresses can still be above the constraint limits $\bar{\sigma}^{s}$ and $\bar{\sigma}^{f}$ as discussed in [15] and [16], but the overall topology of the design is such that local and relatively simple changes, 

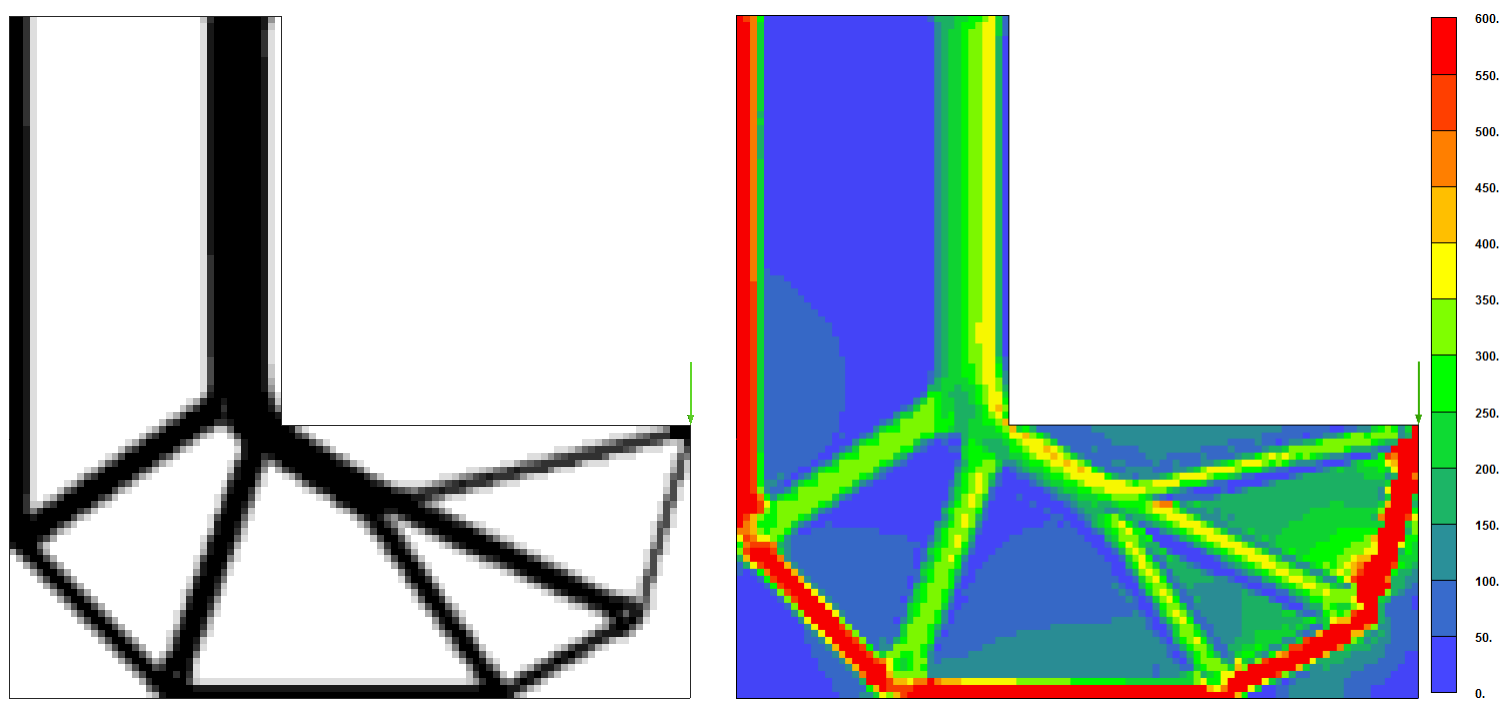

(a) Highest tensile principal stress constraint
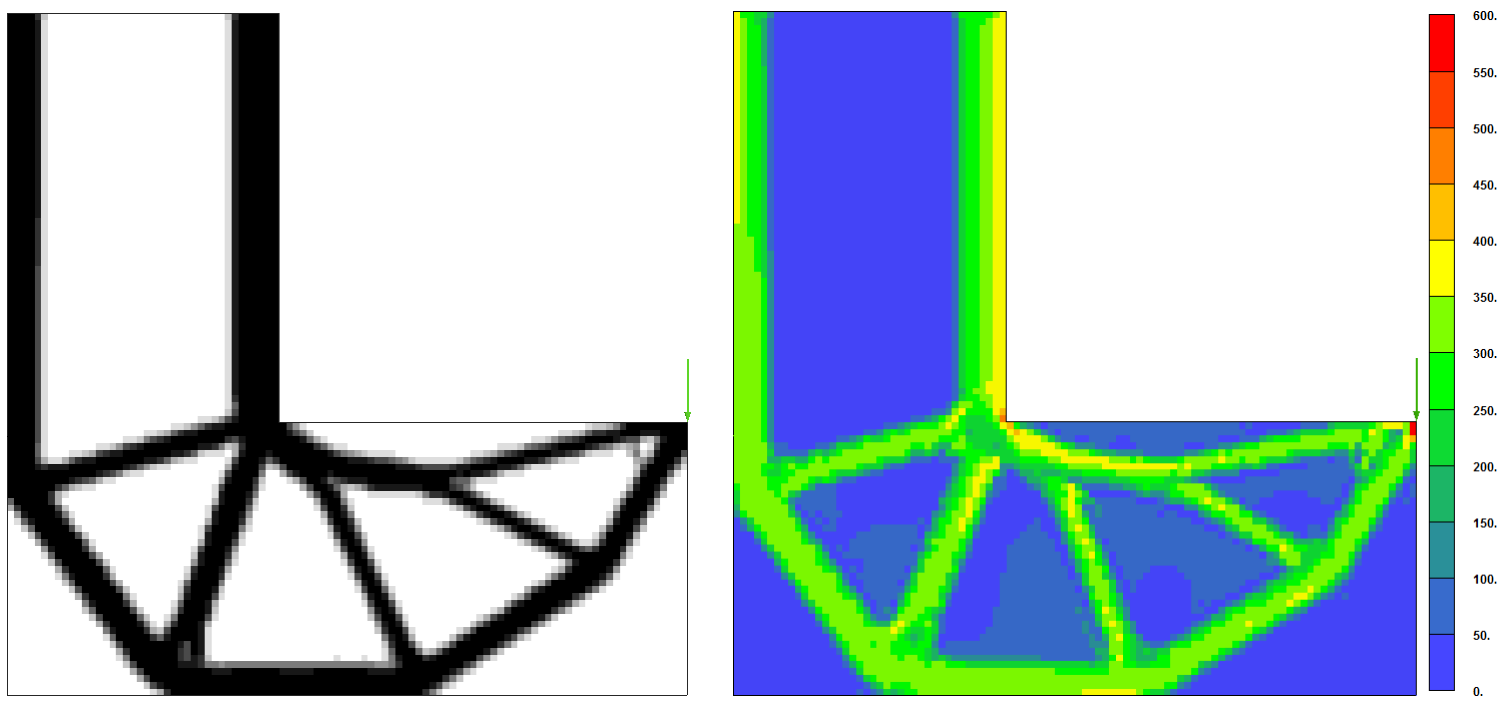

(b) von Mises stress constraint
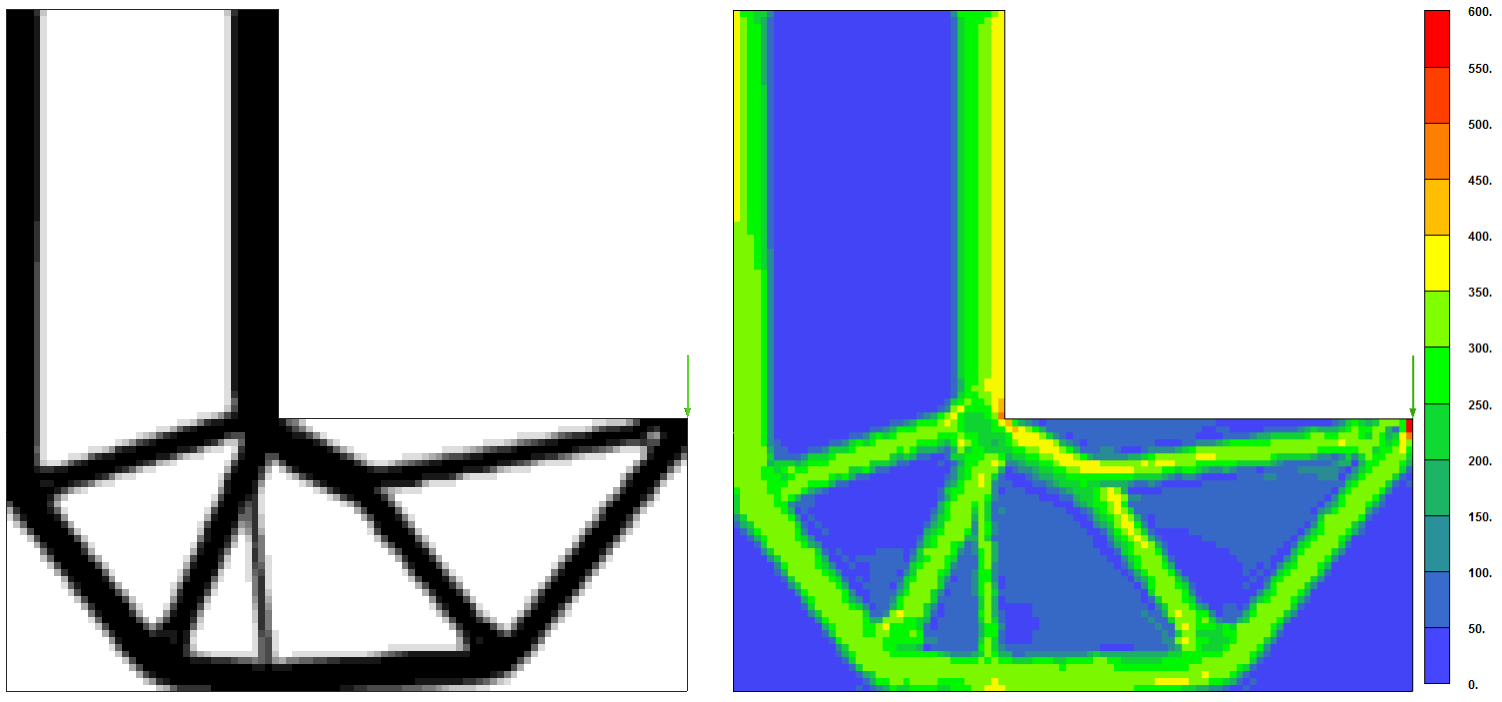

(c) Both highest tensile principal and von Mises stress constraints simultaneously

Fig. 8: Comparison of static stress constraints based on different stress criteria 
such as local size and radii changes, should be sufficient in order to decrease the local stresses.

The paper presents a further step towards more advanced conceptual design optimizations, where constraints are added to simplify the further design work. This gives a more mature design which will save both structural mass and time in the product development process.

\section{Appendix}

Differentiating (13) with respect to design variable $x_{b}$ gives

$\frac{\partial S_{i j}}{\partial x_{b}} \phi_{j}^{\alpha}+S_{i j} \frac{\partial \phi_{j}^{\alpha}}{\partial x_{b}}=\frac{\partial \lambda^{\alpha}}{\partial x_{b}} I_{i j} \phi_{j}^{\alpha}+\lambda^{\alpha} \frac{\partial I_{i j}}{\partial x_{b}} \phi_{j}^{\alpha}+\lambda^{\alpha} I_{i j} \frac{\partial \phi_{j}^{\alpha}}{\partial x_{b}}$.

Using $\frac{\partial I_{i j}}{\partial x_{b}}=0$ and rearranging gives

$\frac{\partial S_{i j}}{\partial x_{b}} \phi_{j}^{\alpha}+\left(S_{i j}-\lambda^{\alpha} I_{i j}\right) \frac{\partial \phi_{j}^{\alpha}}{\partial x_{b}}=\frac{\partial \lambda^{\alpha}}{\partial x_{b}} I_{i j} \phi_{j}^{\alpha}$.

The eigenvector $\phi_{j}^{\alpha}$ is a unit vector, i.e.

$\phi_{i}^{\alpha} I_{i j} \phi_{j}^{\alpha}=\phi_{j}^{\alpha} \phi_{j}^{\alpha}=1$

therefore, if we premultiply $(24)$ by $\phi_{i}^{\alpha}$, we get

$\phi_{i}^{\alpha} \frac{\partial S_{i j}}{\partial x_{b}} \phi_{j}^{\alpha}+\phi_{i}^{\alpha}\left(S_{i j}-\lambda^{\alpha} I_{i j}\right) \frac{\partial \phi_{j}^{\alpha}}{\partial x_{b}}=\phi_{i}^{\alpha} \frac{\partial \lambda^{\alpha}}{\partial x_{b}} I_{i j} \phi_{j}^{\alpha}$,

and rearranging the right hand side of (25) gives

$\phi_{i}^{\alpha} \frac{\partial \lambda^{\alpha}}{\partial x_{b}} I_{i j} \phi_{j}^{\alpha}=\frac{\partial \lambda^{\alpha}}{\partial x_{b}} \phi_{i}^{\alpha} I_{i j} \phi_{j}^{\alpha}=\frac{\partial \lambda^{\alpha}}{\partial x_{b}}$.

As both $S_{i j}$ and $I_{i j}$ are symmetric, we can rewrite as

$\phi_{i}^{\alpha}\left(S_{i j}-\lambda^{\alpha} I_{i j}\right)=\left(S_{j i}-\lambda^{\alpha} I_{j i}\right) \phi_{i}^{\alpha}=0$,

where the zero comes from (13). Combining (25), (26) and (27), we now find that the derivative of the eigenvalues with respect to the design variable reads

$\frac{\partial \lambda^{\alpha}}{\partial x_{b}}=\phi_{i}^{\alpha} \frac{\partial S_{i j}}{\partial x_{b}} \phi_{j}^{\alpha}$

\section{References}

1. Allaire, G., Jouve, F.: Minimum stress optimal design with the level set method. Engineering analysis with boundary elements 32(11), 909-918 (2008)

2. Saab AB. URL http://www.saabgroup.com

3. Bendsøe, M.: Optimal shape design as a material distribution problem. Structural optimization 1(4), 193-202 (1989)

4. Bruggi, M.: On an alternative approach to stress constraints relaxation in topology optimization. Structural and multidisciplinary optimization 36(2), 125-141 (2008)
5. Bruns, T., Tortorelli, D.: Topology optimization of nonlinear elastic structures and compliant mechanisms. Computer Methods in Applied Mechanics and Engineering 190(26-27), 3443-3459 (2001)

6. Cheng, G., Guo, X.: $\varepsilon$-relaxed approach in structural topology optimization. Structural and Multidisciplinary Optimization 13(4), 258-266 (1997)

7. Christensen, P., Klarbring, A.: An introduction to structural optimization, vol. 153. Springer Verlag (2008)

8. Dahlberg, T., Ekberg, A.: Failure, fracture, fatigue: an introduction. Studentlitteratur (2002)

9. Desmorat, B., Desmorat, R.: Topology optimization in damage governed low cycle fatigue. Comptes Rendus Mecanique 336(5), 448-453 (2008)

10. Duysinx, P., Bendsøe, M.: Topology optimization of continuum structures with local stress constraints. International Journal for Numerical Methods in Engineering 43(8), 1453-1478 (1998)

11. Duysinx, P., Sigmund, O.: New developments in handling stress constraints in optimal material distribution. In: 7th AIAA/USAF/NASA/ISSMO symposium on multidisciplinary design optimization, AIAA Paper 98-4906, pp. 1501-1509 (1998)

12. Gruen, F., Eichlseder, W., Puchner, K.: Shape- and topology optimization regarding fatigue analysis. Cumulative Fatigue Damage, Seville (Spain) (2003)

13. Guo, X., Cheng, G., Yamazaki, K.: A new approach for the solution of singular optima in truss topology optimization with stress and local buckling constraints. Structural and Multidisciplinary Optimization 22(5), 364-373 (2001)

14. Haftka, R., Gürdal, Z., Kamat, M.: Elements of structural optimization. Kluwer Academic Publishers (1990)

15. E. Holmberg, B. Torstenfelt, and A. Klarbring.: Stress constrained topology optimization. Structural and Multidisciplinary Optimization, DOI 10.1007/s00158-0120880-7, 1-15, (2013)

16. Holmberg, E., Torstenfelt, B., Klarbring, A.: Global and clustered approaches for stress constrained topology optimization and deactivation of design variables. In: 10th World Congress on Structural and Multidisciplinary Optimization. (2013)

17. Kaya, N., Karen, İ., Öztürk, F.: Re-design of a failed clutch fork using topology and shape optimisation by the response surface method. Materials \& Design 31(6), 3008-3014 (2010)

18. Kirsch, U.: On singular topologies in optimum structural design. Structural and Multidisciplinary Optimization 2(3), 133-142 (1990)

19. Le, C., Norato, J., Bruns, T., Ha, C., Tortorelli, D.: Stress-based topology optimization for continua. Structural and Multidisciplinary Optimization 41(4), 605-620 (2010)

20. Mrzyglod, M., Zielinski, A.: Numerical implementation of multiaxial high-cycle fatigue criterion to structural optimization. Journal of Theoretical and Applied Mechanics 44(3), 691-712 (2006)

21. Mrzyglod, M., Zielinski, A.: Multiaxial high-cycle fatigue constraints in structural optimization. International Journal of Fatigue 29(9), 1920-1926 (2007)

22. Mrzyglod, M., Zielinski, A.: Parametric structural optimization with respect to the multiaxial high-cycle fatigue criterion. Structural and Multidisciplinary Optimization 33(2), 161-171 (2007)

23. MSC Software: MSC Fatigue (2013). URL http://www.mscsoftware.com 
24. Optistruct: 11.0, users manual. Altair engineering. Inc., Troy, MI (2012)

25. París, J., Navarrina, F., Colominas, I., Casteleiro, M.: Topology optimization of continuum structures with local and global stress constraints. Structural and Multidisciplinary Optimization 39(4), 419-437 (2009)

26. París, J., Navarrina, F., Colominas, I., Casteleiro, M.: Block aggregation of stress constraints in topology optimization of structures. Advances in Engineering Software 41(3), 433-441 (2010)

27. Pedersen, N.: Maximization of eigenvalues using topology optimization. Structural and Multidisciplinary Optimization 20(1), 2-11 (2000)

28. Pedersen, N., Nielsen, A.: Optimization of practical trusses with constraints on eigenfrequencies, displacements, stresses, and buckling. Structural and Multidisciplinary Optimization 25(5), 436-445 (2003)

29. Rozvany, G., Birker, T.: On singular topologies in exact layout optimization. Structural and Multidisciplinary Optimization 8(4), 228-235 (1994)

30. Seyranian, A., Lund, E., Olhoff, N.: Multiple eigenvalues in structural optimization problems. Structural and Multidisciplinary Optimization 8(4), 207-227 (1994)

31. Suresh, S.: Fatigue of materials. Cambridge University Press (1998)

32. Svanberg, K.: The method of moving asymptotes - a new method for structural optimization. International journal for numerical methods in engineering 24(2), 359-373 (1987)

33. Svanberg, K.: A class of globally convergent optimization methods based on conservative convex separable approximations. SIAM Journal on Optimization 12(2), 555-573 (2002)

34. Torstenfelt, B.: The TRINITAS project (2013). URL http://www.solid.iei.liu.se/Offered_services/Trinitas

35. Wang, M.Y., Luo, Y.: An enhanced aggregation method for stress-constrained topology optimization problems. In: 10th World Congress on Structural and Multidisciplinary Optimization. (2013)

36. Zhang, W.S., Guo, X., Wang, M.Y., Wei, P.: Optimal topology design of continuum structures with stress concentration alleviation via level set method. International journal for numerical methods in engineering 93(9), 942959 (2013) 